\title{
Análisis bibliométrico de una década (1988-1997) de Espacio Tiempo y Forma. Historia Antigua
}

\author{
Maria teresa Priego Morales *
}

\begin{abstract}
RESUMEN ABSTRACT
En el presente artículo revisamos los primeros diez años de existencia de la revista Espacio Tiempo y Forma. Historia Antigua (1988-1997), inicial foro de expresión de los investigadores vinculados a la UNED. Se analiza el contenido temático de los artículos, la productividad y filiación de los autores, la apertura institucional de la revista asi como, sus aspectos formales y difusión.

In this paper, the first decade of existence of journal "Espacio Tiempo y Forma. Historia antigua" (1988-1997) is analyzed. This journal was initially forum of expression of researchers from the UNED. We analyze the article's topics, productivity and filiation of authors, the journal institutional opening and the format and diffusion aspects.
\end{abstract}

\section{INTRODUCCIÓN}

La revista Espacio, Tiempo y Forma, de la Facultad de Geografia e Historia de la Universidad Nacional de Educación a Distancia, inició su publicación el año 1988, organizada en series, la Serie II. Historia Antigua es la que vamos a estudiar.

La revista, bajo la dirección científica del Departamento de Prehistoria e Historia Antigua nació como un órgano de comunicación de la comunidad

* CSIC. CINDOC, Madrid.

Agradezco a la Dra. Adelaida Román la lectura del borrador de este trabajo, asi como sus sugerencias y consejos 
científica y como medio de difusión de la producción de los profesores tutores y la de los doctores de dicho departamento.

Debido a la particularidad de una universidad como la UNED, con instituciones docentes diseminadas por todo el ámbito nacional y el extranjero, la revista nació abierta a la colaboración nacional e internacional.

En el panorama editorial de la divulgación de las investigaciones sobre Historia Antigua, Espacio, Tiempo y Forma, vino a completar un paisaje realmente pobre. En 1988, en nuestro país sólo se publicaban 4 revistas especializadas. No es que once años después las expectativas sean mucho más halagüeñas, ya que en la actualidad, con una periodicidad regular no se publican más que 6 revistas en las que el peso de la Historia Antigua sea preferente.

Aunque la Serie II de la revista objeto de nuestro estudio tiene como epígrafe "Historia Antigua», como veremos más adelante, acoge trabajos propios de otras disciplinas que tienen sus propios órganos científicos de expresión.

La evaluación de una revista es complicada y puede abordarse desde muy diferentes perspectivas, siendo una de ellas la cuantitativa. El análisis bibliométrico es uno de los instrumentos más útiles a este respecto. A partir de unos parámetros previamente seleccionados (contenidos, autores, estudio formal) se logra una pormenorizada descripción de la revista, cuya interpretación permitirá definir su especificidad, la trayectoria experimentada por el campo disciplinar, así como las relaciones entre los científicos.

\section{METODOLOGIA}

Hemos seleccionado para nuestro trabajo, sólo los artículos científicos, sin entrar a estudiar otras secciones de la revista como editoriales, recensiones, noticias, etc.

El trabajo lo dividiremos en tres partes: 1) estudio del contenido de los artículos. 2) estudio de los autores (productividad, filiación). 3) estudio de la revista (productividad, aspectos formales, apertura institucional, uso y difusión).

El método de análisis de contenido que hemos seguido entraña serias dificultades y ciertos riesgos, porque hemos preferido asignar opciones fijas y determinadas para definir el contenido temático de cada artículo sin ambivalencias, para situarlo en un marco muy concreto y definido, asignándole un único lugar, aquel que precisamente expresa su contenido más 
específico independientemente de que éste sea susceptible de ser asignado a distintos campos temáticos.

Para su clasificación temática hemos escogido la de la Base de Datos ISOC: historia política, historia militar, historia económica, historia social, historia de las religiones, cultura y modo de vida, ciencia y técnica, administración e instituciones, e historia urbana.

\section{ANÁLISIS DEL CONTENIDO DE LOS ARTÍCULOS}

En los diez años que vamos a estudiar, 1988-1997 ambos inclusive, se han publicado 203 artículos, de los cuales el $52,7 \%$ son de Historia Antigua, el $36 \%$ son de Arqueología, y el $10,3 \%$ son de diferentes áreas temáticas que van desde la Historia Medieval, Arte, Geografia, etc.

En la figura 1 se recogen estos datos pormenorizados por años.

Parece claro que a partir de 1990 se consolida una preferencia o mayor dedicación por la Historia Antigua, que parece haberse equilibrado en 1997.

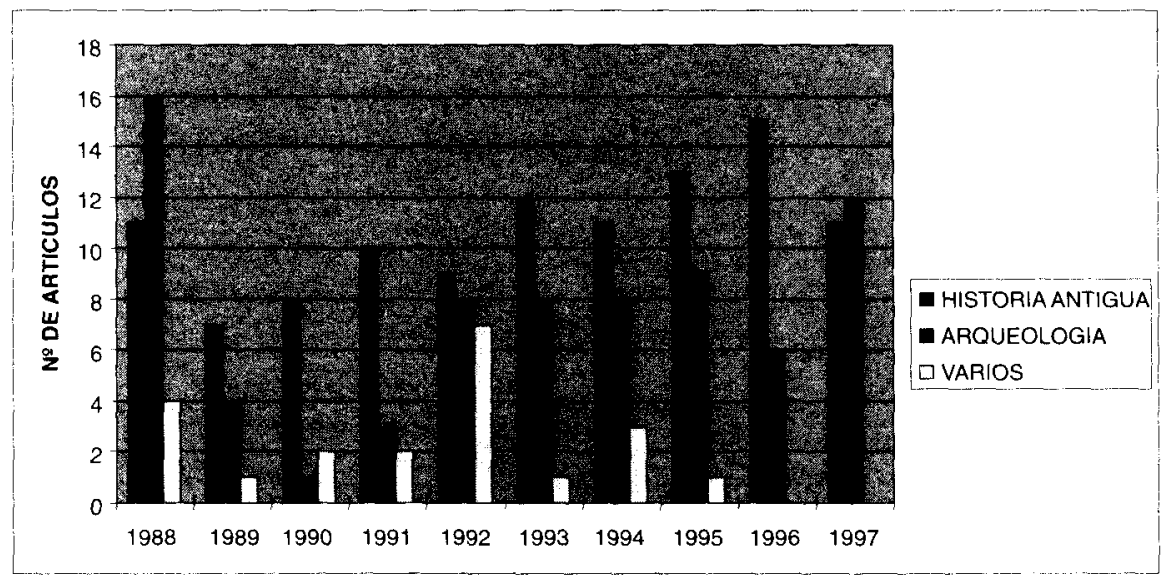

Fig. 1.

De los 107 artículos de Historia Antigua, el 64,45\% son de Historia Antigua Universal. La Historia Antigua de España, representa un $35,55 \%$ de estos trabajos.

En la figura 2 se recogen distribuidos por áreas temáticas los 69 artículos de Historia Antigua Universal. Como puede observarse es la historia de las religiones, el tema más estudiado por los científicos que han publicado en esta revista. El tema que menos interés ha suscitado la historia militar. 


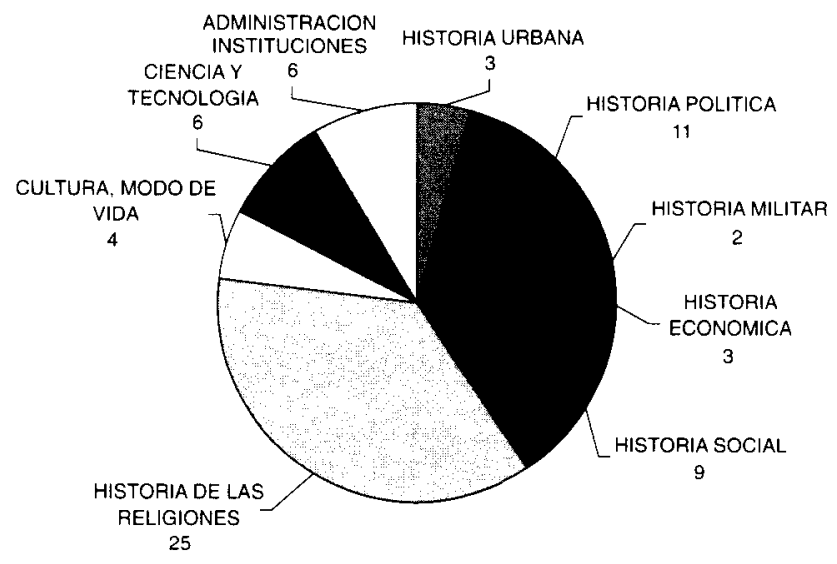

Fig. 2. Historia Antigua Universal.

De los 38 trabajos de Historia Antigua de España, el tema más estudiado es la historia de las religiones con un 26,3 de los artículos. Tres áreas temáticas: historia política, cultura y modo de vida, y administración e instituciones, han suscitado el mismo interés de los investigadores, publicándose 2 trabajos de cada una de ellas. La figura 3 muestra los datos de cada una de las clasificaciones temáticas.

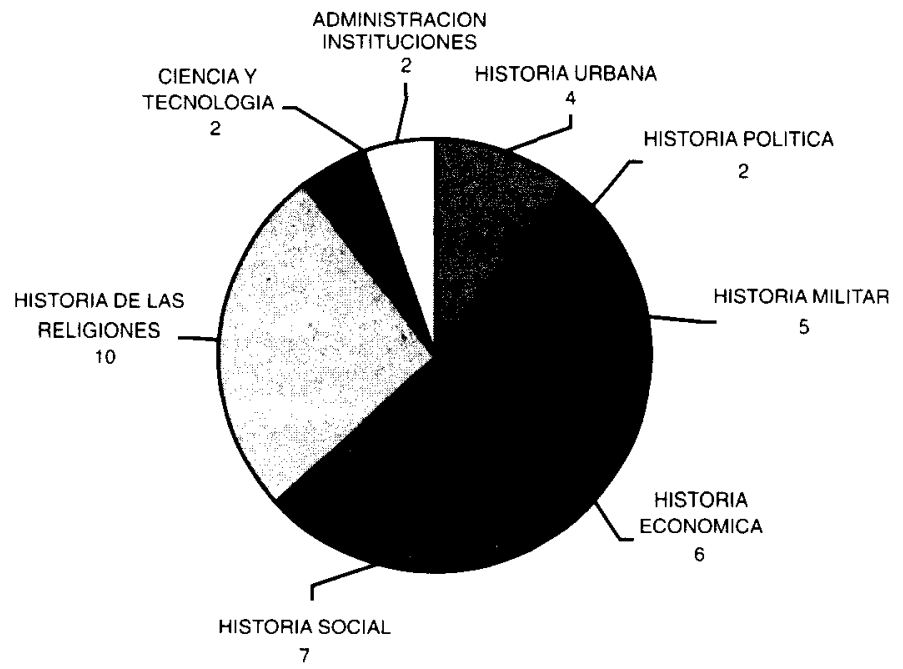

Fig. 3. Historia Antigua España. 
De los 75 trabajos que se han clasificado como de Arqueologia, y siguiendo así mismo la clasificación de la Base de Datos ISOC, estos artículos se han atribuido a las épocas y culturas que se muestran en la figura 4. Como puede observarse, la época romana o paleocristiana en la Península Ibérica es el tema más publicado con el $48 \%$ de los trabajos, seguido de la Edad del Hierro y el mundo ibérico con el 22,6\%. En cuanto a países extranjeros, la cultura egipcia es la que más interés ha suscitado a nuestros investigadores.

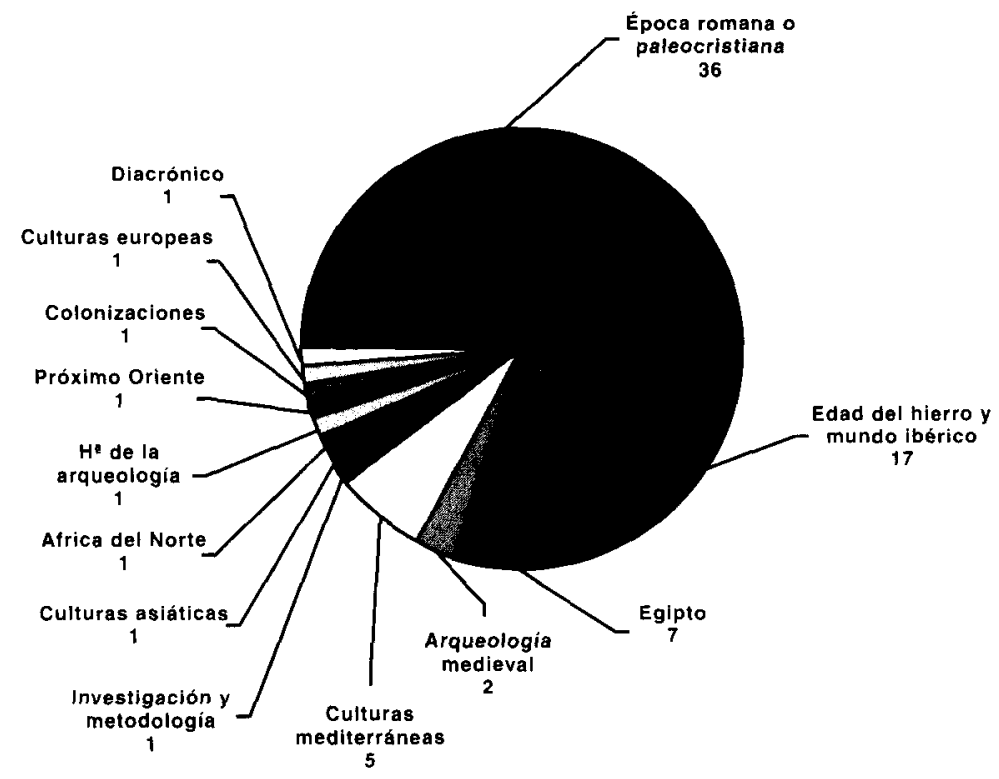

Fig. 4. Arqueología.

\section{ANALISIS DE LOS AUTORES}

a) Nivel de colaboración de los autores de los trabajos

De los 203 artículos publicados, 159 eran publicados por un sólo autor, el $78,3 \%$ de los trabajos, y sólo el $21,7 \%$ son trabajos realizados en colaboración. 39 han sido realizados por 2 autores, $19,2 \%$, por 3 autores hay 3 trabajos y por 4 autores, 2 . Por tanto la tendencia al trabajo aislado es patente.

En la figura 5 se muestra el número de autores por año en relación con el número de artículos año. Los 203 artículos han sido realizados por 254 firmas. 


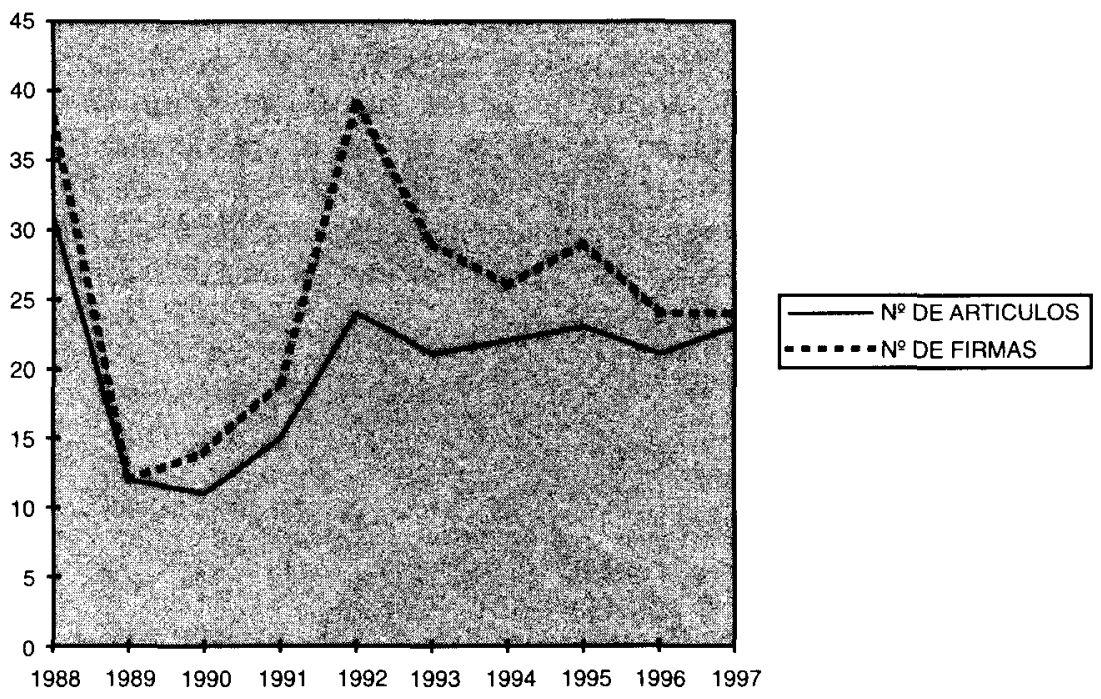

Fig. 5.

\section{b) Media de firmas-trabajo}

Para la elaboración de los 203 artículos publicados en estos diez años, se ha contado con la participación de 146 autores diferentes, contabilizándose para el conjunto de éstos un total de 254 firmas.

Si se divide el número de autores entre el número de trabajos se obtiene la media de autores por trabajo que en el caso que se analiza es de 1,25 , bastante baja, e indicativa del escaso nivel de colaboración que antes se mencionaba.

\section{c) Productividad de los autores}

No todos los autores han participado con igual frecuencia en la revista. En la tabla 1 se recogen estas diferencias. En ella queda patente que el $64,38 \%$ de los autores lo han hecho de una manera ocasional.

Los autores más productivos de la revista se recogen en la tabla 2; se incluyen los que han publicado cinco o más trabajos y su filiación. 
Tabla 1.

\begin{tabular}{cccc}
\hline$N^{\circ}$ de Artículos & Autores & $\%$ \\
\hline 1 & 94 & $64,38 \%$ \\
2 & 30 & $20,54 \%$ \\
3 & 8 & $5,47 \%$ \\
4 & 6 & $4,18 \%$ \\
5 & 5 & $3,42 \%$ \\
6 & - & - \\
7 & - & $0,68 \%$ \\
8 & 1 & $0,68 \%$ \\
9 & - & - \\
10 & 1 & $0,68 \%$ \\
\hline TOTAL & 11 & 146 & $99,5 \%$ \\
\hline
\end{tabular}

Como puede observarse, sólo 8 autores han publicado cinco o más trabajos. De ellos, los dos más productivos son autores que desarrollan sus investigaciones en la UNED, pero, sólo uno de ellos, la Dra. Pilar Fernández Uriel, pertenece en la actualidad al Comité de Redacción de la revista. Hay dos autores que han publicado 5 trabajos pero no hemos hecho constar su filiación, porque ha ido cambiando en el transcurso del tiempo.

Tabla 2.

\begin{tabular}{lcc}
\hline & $N^{\circ}{ }^{\circ}$ de Trabajos & Centro \\
\hline Vázquez Hoys, Ana María & 11 & UNED \\
Fernández Uriel, Pilar & 9 & UNED \\
García-Gelabert Pérez, María Paz & 7 & UCM \\
Abad Varela, Manuel & 5 & UNED \\
Burgaleta Mezo, Francisco Javier & 5 & - \\
Hoyo Calleja, Javier del & 5 & UAM \\
López Monteagudo, Guadalupe & 5 & CSIC \\
Vidal Manzanares, César & 5 & - \\
\hline
\end{tabular}

ANALISIS DE LA REVISTA

Desde 1988 hasta 1997, este es el período objeto de nuestro análisis como hemos indicado anteriormente, se han publicado 203 artículos, por tanto la media es de 20,3 artículos por año. El año más productivo fue el de su nacimiento con 31 artículos y el menos 1990 con 11. En la figura 1 
se recogen los artículos publicados cada año.

La serie comenzó con mucha aceptación publicándose en su primer número como hemos indicado 31 trabajos, luego en los tres años siguientes publica 12, 11 y 15 trabajos respectivamente, a partir de 1992 parece que la situación se estabiliza y se publican todos los años más o menos el mismo número de artículos, oscilando entre 24 en el año 1992 y 21 en los años 1993 y 1996.

Al ser una revista española la mayoría de los trabajos están en español, el $93,1 \%$. El 6,8\% restantes están en otras lenguas: 8 están en francés, 3 en catalán, 2 en portugués y 1 en inglés.

\section{a) Apertura institucional de la revista}

De las 254 firmas que han elaborado los 203 trabajos objeto de nuestro estudio, sólo 189 han hecho constar su filiación, lo que supone un $74,8 \%$ del total. Este porcentaje, sin ser completamente satisfactorio es superior al de las áreas de Humanidades y Ciencias Sociales si se exceptúa la Psicología.

De los 189 trabajos, el $92,6 \%$ han sido efectuados en instituciones españolas, y el $7,4 \%$ restantes en centros extranjeros. Debemos destacar que la universidad editora de la revista, aporta el 30,1\% de los artículos.

Dentro de las instituciones españolas y haciendo una tipología institucional, estos son los porcentajes de presencia:

Universidades españolas $77,2 \%$

C.S.I.C. $3,7 \%$

Museos $3,7 \%$

Otros centros de Investigacion. $7,9 \%$

En las tablas 3 y 4 se recogen estos datos pormenorizados. 
Tabla 3.

\begin{tabular}{lc}
\multicolumn{1}{c}{ Universidades Españolas } & N. ${ }^{\circ}$ de \\
\hline & 57 \\
UNED & 26 \\
Universidad Autónoma de Madrid & 12 \\
Universidad Complutense de Madrid & 9 \\
Universidad de Valladolid & 5 \\
Universidad Autónoma de Barcelona & 5 \\
Universidad de Extremadura & 5 \\
Universidad de Murcia & 5 \\
Universidad de Santiago de Compostela & 3 \\
Universidad de Alcalá de Henares & 3 \\
Universidad de Córdoba & 3 \\
Universidad de Oviedo & 3 \\
Universidad de Valencia & 2 \\
Universidad de Alicante & 2 \\
Universidad de Barcelona & 2 \\
Universidad de Cantabria & 2 \\
Universidad de la Laguna & 1 \\
Universidad deAlmería & 1 \\
Universidad de Castilla-La Mancha & \\
\hline
\end{tabular}

Tabla 4.

\begin{tabular}{ll}
\multicolumn{1}{c}{ Centros Españoles } & N. ${ }^{\circ}$ de Trabajos \\
\hline Consejo Superior de Investigaciones Científicas & 7 \\
Asociación Española de Amigos de la Arqueología & 4 \\
Instituto de Bachillerato Beatriz Galindo & 2 \\
Instituto de Salud Carlos III & 2 \\
Museo Arqueológico de Elda & 2 \\
Museo de Navarra & 2 \\
Diputación de Barcelona. Servicio Patrimonio Arqueológico & 1 \\
Escuela Técnica Superior de Arquitectura. Barcelona & 1 \\
Institut Estudis Catalans & 1 \\
Museo Arqueológico de Barcelona & 1 \\
Museo Arqueológico de Orense & 1 \\
Museo Arqueológico de Sevilla & 1 \\
Museo de Lugo & 1 \\
Real Academia de la Historia & 1 \\
Servei d'Arqueologia de Barcelona & 1 \\
Servicio de Investigaciones Arqueológicas y Prehistóricas - Castellón & 1 \\
\hline
\end{tabular}

En la tabla 5 se recogen los Centros extranjeros cuyos investigadores han colaborado con la revista. Son 14 firmas, lo que representa un $7,4 \%$ del total de autores que han hecho constar su filiación. 
Como se puede apreciar, todos son centros europeos, no hay colaboradores de fuera de nuestro continente. Es interesante destacar que las firmas que más han participado son autores que pertenecen a universidades y centros de investigación franceses, con un $4,7 \%$ del total.

Tabla 5.

\begin{tabular}{lc}
\hline Universidad y Centros de Investigación Extranjeros & N. ${ }^{\circ}$ de \\
\hline Universite de Lyon & 3 \\
CNRS. Paris & 2 \\
Centre Pierre Paris & 1 \\
Centro Estudios Históricos de Leningrado & 1 \\
CNRS. Aix-en-Provence & 1 \\
Instituto Portugués do Patrimonio Cultural & 1 \\
Kommission Altegeschichte und Epigraph'k. Munich & 1 \\
Universidade de Koimbra & 1 \\
Université Haut-Bretagne-Rennes & 1 \\
Université de Provence & 1 \\
University of London & 1 \\
\hline
\end{tabular}

A tenor de estas cifras podemos concluir diciendo que la apertura institucional es clara, ya que el $69,9 \%$ de los trabajos han sido efectuados por investigadores no vinculados a la UNED.

\section{b) Aspectos formales}

Periodicidad: la revista debe indicar su periodicidad y cumplirla, la revista objeto de nuestro análisis, no incluye ese dato, pero es una revista anual, que cumple con un ligero retraso su publicación.

Datos de identificación: En cubierta y portada, las publicaciones periódicas deben incluir, título de la revista, la entidad editora, el año de publicación, volumen, tomo, serie e ISSN. Debe incluir sumario y en los artículos sus datos de identificación, esto es el nombre del autor del trabajo, su título, debe constar así mismo el nombre de la revista y las páginas que ocupa el trabajo. Estas normas las cumple parcialmente Espacio Tiempo y Forma. Historia Antigua.

Organos de gestión: los órganos de gestión de la revista objeto de nuestro análisis están incluidos en cubierta. Tiene director, secretario y Comité de Redacción y con posterioridad se incluye un Consejo Asesor.

Normas de publicación de artículos en revistas científicas: Dentro de estas normas debemos destacar la inclusión de resúmenes tanto en espa- 
ñol como en inglés, las palabras clave, y los lugares de filiación de los autores. Todos estos datos no están recogidos en la revista que analizamos.

\section{c) Difusión}

Para el análisis de la difusión de la revista podriamos haber utilizado diversos mecanismos. Hemos optado por la consulta a los catálogos colectivos más importantes para conocer su presencia en las Bibliotecas.

Recurriendo tanto a medios automatizados (OCLC, Catalogo Colectivo de Publicaciones periódicas, REBIUN, etc.) como manuales, hemos localizado la revista en 15 bibliotecas españolas y 3 extranjeras.

\section{CONCLUSIONES}

Las publicaciones periódicas se consideran, entre los vehículos de comunicación científica, la principal fuente de información sobre el desarrollo de una disciplina, por eso la consolidación de una revista como Espacio Tiempo y Forma. Historia Antigua, con el panorama tan poco alentador como nos referíamos al principio, nos informa de la pujanza que esta especialidad dentro de la Historia, esta cobrando en la actualidad.

Dos son los aspectos que de este análisis queremos destacar: primero, la apertura institucional de la revista, y segundo que el $64,38 \%$ de los autores hayan publicado un solo artículo, esto también hace referencia a esa apertura, ya que han dado oportunidad a historiadores que inician su carrera investigadora.

Alentamos a los responsables de la revista a continuar en la línea abierta en el último número objeto de nuestro análisis, ya que en él recogen muchas de las normas que hemos comentado anteriormente. Subrayamos la importancia de que todos los artículos tengan resumen en español e inglés, muy interesante si queremos que nuestra bibliografía se difunda en el extranjero.

\section{BIBLIOGRAFIA}

Alaez Fernandez, Máximo. Estudio bibliométrico de los trabajos aparecidos en Clínica y Salud (1990-1997). Clínica y Salud, 1998, 9, (2), 509-518.

Almuñza Fernández, Celso, et al. "Hispania», revista de historia (1940-1989). Análisis y evolución de contenidos. Hispania, 1990, (175), 393-416.

CREspo Ortiz dE ZaRATE, Santos. La aportación historiográfica de Hispania Antiqva a la historia de la antigüedad (1971-1995). Hispania Antiqva, 1995, 19, 397-425. 
Rodriguez Alcalde, Angel; San Millan Bujanda, María Jesus; Sánchez Nistal, José María; Chapa Brunet, Teresa; Martinez Navarrete, Maria Isabel; Ruiz Zapatero, Gonzalo. Análisis bibliométrico de Trabajos de Prehistoria: un chequeo a la prehistoria española de la tres últimas décadas. Trabajos de Prehistoria, 1993, 50, 11-37.

RiviRA LloRENS, Salvador. Estudio bibliométrico del Boletín de la Asociación de Amigos de la Arqueología. Boletín de la Asociación de Amigos de la Arqueología, 199 , 57-65.

SÁnCHEZ HeRnÁndez, Antonio; Quiñones VIDAL Elena; Cameo Olcina, Mercedes. La revista Anales de Psicologia desde un punto de vista historigráfico: la primera década de una publicación con raices académicas (1984-1994). Anales de Psicología, 1995, 11 (1), 1-18. 